\title{
Oportunidades comerciales para el aceite de oliva de Castilla-La Mancha
}

\author{
Por Rodolfo Bernabéu, ${ }^{a, b, \star}$ Miguel Olmeda, ${ }^{a, b}$ Mónica Díaz ${ }^{b}$ y Raquel Olivas ${ }^{b}$ \\ ${ }^{a}$ E.T.S. Ingenieros Agrónomos de Albacete. Universidad de Castilla-La Mancha \\ Campus Universitario s/n, 02071 Albacete (España). \\ ${ }^{b}$ Instituto de Desarrollo Regional. Universidad de Castilla-La Mancha \\ Campus Universitario s/n, 02071 Albacete (España) \\ ( ${ }^{\star}$ Autor para correspondencia: Rodolfo.Bernabeu@ $@$ uclm.es)
}

\section{RESUMEN}

Oportunidades comerciales para el aceite de oliva de Castilla-La Mancha.

El aceite de oliva producido en Castilla-La Mancha tiene una difícil comercialización tanto por el aumento de la competitividad nacional como internacional así como por su fácil sustitución por otros aceites de semillas más baratos. Para incrementar su competitividad, resulta necesario conocer cuáles son las preferencias de los consumidores. Para ello, se realizaron 420 encuestas en diciembre de 2006 a consumidores de aceite en el área metropolitana de Madrid. Mediante las técnicas de análisis conjunto, simulación y logit, se ha detectado que el tipo de aceite preferido por los consumidores es el de oliva virgen extra, que parecen existir oportunidades de mercado para los producidos ecológicamente en Castilla-La Mancha y que la máxima disposición al pago por un aceite de oliva ecológico, respecto a otro convencional, es del $13,1 \%$. Adicionalmente a lo anterior y con el objetivo de favorecer la comercialización del aceite de oliva ecológico producido en Castilla-La Mancha, la Administración regional podría promover la creación de la Indicación Geográfica Protegida de Aceites de Oliva Ecológicos de Castilla-La Mancha.

PALABRAS CLAVE: Análisis Conjunto - Comportamiento consumidor - Disposición máxima al pago - Marketing agroalimentario - Segmentación.

\section{SUMMARY}

Commercial opportunities for olive oil from CastillaLa Mancha (Spain).

Quality olive oils produced in Castilla-La Mancha are difficult to commercialize in the national and international markets due to increasing competition among olive oil companies and their easy replacement by other cheaper seed oils. To increase competitiveness, consumer preference must be discovered. Therefore, 420 surveys were made on vegetable oil consumers in the Madrid metropolitan area in December, 2006. It was detected by means of the conjoint analysis technique, simulation and analysis logit that the type of vegetable oil preferred by consumers was the extra virgin olive oil. Some market opportunities seem to exist for the organic olive oil produced in Castilla-La Mancha and consumers' maximum willingness to pay for organic olive oil with regard to the conventional oil is $13.1 \%$. In addition to this, and with the aim of contributing to the commercialization of the organic olive oil produced in Castilla-La Mancha, the Regional Government of Castilla-La Mancha (Spain) could promote the creation of the Protected
Geographical Indication of Castilla-La Mancha Organic Olive Oils.

KEY-WORDS: Conjoint Analysis - Consumer behavior Food Marketing - Segmentation - Willingness to pay.

\section{INTRODUCCIÓN}

Las exigencias de los consumidores hacia productos preferentemente nuevos, de calidad y con garantías sanitarias, está provocando una adaptación de las empresas en general y las elaboradoras de aceite en particular, para satisfacer estas demandas a la vez que intentan diferenciarse de la competencia y así poder competir en el mercado.

En este sentido, la competencia empresarial en la producción de aceites hay que contextualizarla en el mercado de las materias grasas, ya que aceites y grasas son, en cierta medida, productos sustitutivos entre sí. A su vez, dentro de la producción de aceites, la competencia principal está entre los aceites procedentes de oliva y los de semillas.

En España, desde el punto de vista de la producción de aceites, las diferencias en los costes de producción derivados de la diversa naturaleza agrícola de la materia prima (cultivo intensivo frente a extensivo) y la localización geográfica, determina una estructura de precios relativos entre los aceites de oliva y los aceites de semillas, que es francamente desfavorable para el consumo de los primeros.

Adicionalmente, la elaboración de aceites cuenta con la particularidad de que su comercialización se encuentra en creciente competencia, tanto en el ámbito nacional (debido a la proliferación de marcas individuales y colectivas), como internacional.

Debido a estas dos características básicas, las empresas elaboradoras de aceite tienen que crearse una imagen diferenciada de las de sus competidores, mediante la creación de marcas fuertes, a la vez que adaptarse a las necesidades y/o deseos de los consumidores, para así lograr ventajas competitivas.

En este proceso de adaptación, resulta necesario desarrollar unas estrategias de incremento del consumo de aceite de oliva mediante el sosteni- 
miento de una adecuada relación de precios con otros productos parcialmente sustitutivos, lo cual sólo puede ser posible debido al prestigio que gozan los aceites de oliva por distintas razones dietéticas y de salud, así como pertenecer al grupo de alimentos integrantes en la denominada dieta mediterránea.

Por una parte, desde el punto de vista del consumo $^{1}$, el consumidor cada vez está más interesado por conocer las cualidades del producto que va a comprar (Sanz et al., 1997). Es este deseo del consumidor de estar informado sobre lo que va a comprar lo que obliga a los productores a diferenciar sus mercancías de las de los competidores.

Por otra parte, desde un punto de vista comercial, se puede establecer como elemento diferenciador del aceite su carácter ecológico (Brugarolas y Rivera, 2001), ya que los alimentos ecológicos tienen una imagen positiva para los consumidores (Mann, 2003) y se diferencian fundamentalmente por su carácter saludable y respetuoso con el medio ambiente (Aguirre et al., 2003; Sanjuán et al., 2003; Chang y Zepeda, 2004; Armstrong et al. 2005; De Boer et al., 2006).

En este sentido, la superficie española de agricultura ecológica en 2007 fue de 988.323 ha, de las cuales 94.251 ha se dedicaron al olivar (MAPA, 2008), lo que supone el $9,5 \%$ de la superficie ecológica nacional. En dicho año, en Castilla-La Mancha, se cultivaron 48.452 ha de agricultura ecológica (lo que supone el $4,9 \%$ de la superficie nacional), de las cuales se dedicaron 7.651 ha al olivar ecológico (lo que supone el $15,8 \%$ de la superficie ecológica de la Comunidad y a su vez, el $8,1 \%$ de la superficie ecológica de olivar nacional) (MAPA, 2008).

No obstante, a pesar de la superficie inscrita en agricultura ecológica en Castilla-La Mancha, el consumo de alimentos ecológicos en la región es muy bajo. Con datos de 2004, Bernabéu y Olmeda (2007) estimaron que los consumidores habituales de alimentos ecológicos en Castilla-La Mancha oscilaban entre el $2 \%$ y el $5,8 \%$. Por otra parte, y para ese mismo año, Noomene (2005) estimó en un $8,9 \%$ los consumidores habituales de alimentos ecológicos en Madrid (lo que además constituyó un máximo nacional), además de ser un centro de mucha vida social y un buen escaparate para lanzar nuevos productos agroalimentarios.

Por lo anterior, Castilla-La Mancha se configura como una región productora de alimentos ecológicos a la vez que poco consumidora de los mismos. Además, dentro de la producción de alimentos ecológicos en Castilla-La Mancha, la producción de aceite de oliva ecológico podría resultar relevante no sólo por la superficie dedicada al olivar, sino también por el saber hacer y la experiencia (knowhow) de los olivareros y empresarios regionales en la producción de aceite de oliva en general.
En este sentido, el presente trabajo tiene como objetivo básico analizar cuáles podrían ser las oportunidades comerciales para las empresas productoras de aceite de oliva en Castilla-La Mancha para favorecer su consumo en el mercado de Madrid así como comprobar cuál es la oportunidad actual de ofrecer un aceite de oliva ecológico. Para lograr dicho objetivo, resulta necesario determinar; a) la estructura de preferencias del consumidor de aceite, b) la cuota de mercado estimada del aceite oliva ecológico producido en Castilla-La Mancha y c) la máxima disposición al pago que tienen los consumidores por un aceite ecológico respecto a otro convencional.

\section{MATERIALES Y MÉTODOS}

En este trabajo se encuestó a 420 consumidores de aceite del mercado de Madrid (capital y área metropolitana), que se disponían a comprar alimentos para su consumo en el hogar, durante el mes de diciembre de 2006. Para el diseño de la muestra se utilizaron los datos de población de la Comunidad de Madrid del año 2005 según el Servicio de Estadísticas de la Comunidad de Madrid (IECM, 2006). El muestreo se realizó de forma aleatoria y estratificada con afijación proporcional por población, género y edad (entre 18 y 24 años, 25 y 34 años, 35 y 49 años, 50 y 64 años y más de 64 años), para un nivel de error inferior al $5,0 \%$ y un nivel de confianza al $95,5 \%(p=q=0,5 ; k=2)$ (Tabla 1). Previo al trabajo de campo se realizó un cuestionario prueba a 25 personas para verificar la redacción y comprensión de las preguntas (Rivera, 1989).

La distribución de encuestas se realizó en el entorno de tres hipermercados (Alcampo, Carrefour e Hipercor) y en el Mercado de Las Ventas, ya que, según el Comité de Agricultura Ecológica de la Comunidad de Madrid, éstos son los principales establecimientos de compra de los consumidores de alimentos ecológicos.

Tabla 1

\section{Ficha técnica de la encuesta}

\begin{tabular}{|c|c|}
\hline Ámbito & Comunidad de Madrid \\
\hline Universo & $\begin{array}{l}\text { Consumidores de aceite } \\
\text { mayores de edad. }\end{array}$ \\
\hline Tamaño muestral & 420 encuestas. \\
\hline Error muestral & $\pm 5,0 \%$ \\
\hline Nivel de confianza & $95,5 \%(p=q=0,5 ; k=2)$ \\
\hline Muestreo & $\begin{array}{l}\text { Aleatorio estratificado con } \\
\text { afijación proporcional a la } \\
\text { población, al genero y a la } \\
\text { edad }\end{array}$ \\
\hline Control & De coherencia y estabilidad \\
\hline Cuestionario previo & 25 personas \\
\hline Trabajo de campo & Diciembre de 2006 \\
\hline
\end{tabular}

${ }^{1}$ En España, según el Panel Alimentario (MAPA, 2007), en el año 2006 el consumo de todos los aceites fue de 20,63 L/persona, siendo el aceite más consumido el de oliva (8,46 L/persona), seguido del aceite de girasol (7,35 L/persona), del aceite de oliva virgen (3,68 L/persona), del aceite de orujo $(0,50 \mathrm{~L} /$ persona) y otros aceites $(0,65 \mathrm{~L} /$ persona $)$. 
Las encuestas se realizaron en diez centros distintos, siete de ellos se localizaban en Madrid capital (Moratalaz, Fuencarral, Arganzuela, Hortaleza, Latina, San Blas y Ventas) y tres en municipios de alrededor (Leganés, Pozuelo y Alcobendas).

El cuestionario definitivo, dirigido tanto a consumidores como a potenciales consumidores de alimentos ecológicos (es decir, aquellos que no son consumidores actuales de alimentos ecológicos pero manifiestan una predisposición favorable a su consumo en el futuro), estaba estructurado y dividido en cinco apartados: características de consumo de alimentos ecológicos, disposición máxima al pago de los alimentos ecológicos (respecto a los convencionales), actitudes de compra de alimentos, cuestiones sobre los estilos de vida y características socioeconómicas del consumidor. Dentro de las preguntas relativas a las actitudes en la compra de alimentos, se incluyeron las encaminadas para detectar la estructura de preferencias del consumidor.

La técnica utilizada para determinar las preferencias del consumidor, ha sido la de Análisis Conjunto (Green y Rao, 1971), en los que el sujeto informa de la preferencia global del perfil de un producto, estimando el investigador la importancia de cada uno de los atributos en la percepción global del sujeto.

A través de la bibliografía existente, entrevistas con expertos de diversos ámbitos (empresarial, institucional e investigador) $^{2}$ y de un cuestionario previo se han seleccionado los atributos y niveles más representativos del proceso de compra de aceite para su consumo en el hogar. Los atributos identificados como más importantes en el proceso de compra de aceite han sido: el precio de la botella de $1 \mathrm{~L}(1,2 €, 4 € \text { y } 5 €)^{3}$, el tipo (girasol, oliva y oliva virgen extra), el origen (Castilla-La Mancha, nacional y extranjero) y, finalmente, el sistema de producción (ecológico y convencional). Con estos cuatro atributos y sus once niveles se han obtenido $54(3 \times 3 \times 3 \times 2)$ posibles productos.
Evaluar los 54 posibles productos sería prácticamente inviable para una persona. Sin embargo, mediante un diseño factorial fraccionado, por el que permite que no exista correlación entre los atributos (diseño ortogonal), a través del procedimiento Orthoplan del módulo Categories (SPSS, 2008), ha permitido reducir las combinaciones a nueve (Tabla 2). La elección de un diseño ortogonal frente a la presentación de todas las combinaciones posibles de productos limita la obtención de información únicamente a los efectos principales de los atributos, eliminando las interacciones (ya que éstas explican menos del $10 \%$ sobre el total de las preferencias), a la vez que presenta la ventaja de poder ofrecer sólo nueve productos a cada encuestado, estimándose que esta ventaja supera a dicho inconveniente (Kirk, 1982; Braña et al., 1995).

Una vez diseñadas las tarjetas de los aceites hipotéticos, éstas se presentaron a cada uno de los encuestados (Anexo 1), quienes asignaron una puntuación de 1 a 10 a cada tarjeta en función de sus preferencias declaradas, con posibilidad de repetir puntuación en más de una tarjeta, correspondiendo el 1 al menor grado de preferencia y el 10 al mayor grado de preferencia (método de perfil completo).

\section{Anexo 1}

Ejemplo de modelo de tarjeta presentada a los consumidores

Puntúe, de 1 (menos preferido) a 10 (más preferido), su intención de compra de un aceite en su establecimiento habitual, con las siguientes características:

\begin{tabular}{lll}
\hline Precio: & $4 €$ & \\
Tipo: & Oliva & \\
Origen: & Castilla-La Mancha & \\
Sistema: & Convencional & Puntuación: \\
& & \\
\hline
\end{tabular}

Tabla 2

Tarjetas de aceites hipotéticos expuestos a los encuestados

\begin{tabular}{ccccc}
\hline № Tarjeta & Precio $^{\mathbf{a}}$ & Tipo & Origen & Sistema \\
\hline 1 & $5 €$ & Oliva & Extranjero & Convencional \\
2 & $5 €$ & Girasol & Castilla-La Mancha & Ecológico \\
3 & $4 €$ & Oliva Virgen Extra & Extranjero & Ecológico \\
4 & $4 €$ & Girasol & Nacional & Convencional \\
5 & $4 €$ & Oliva & Castilla-La Mancha & Convencional \\
6 & $1,2 €$ & Girasol & Extranjero & Convencional \\
7 & $1,2 €$ & Oliva Virgen Extra & Castilla-La Mancha & Convencional \\
8 & $5 €$ & Oliva Virgen Extra & Nacional & Ecológico \\
9 & $1,2 €$ & Oliva & Nacional & \\
\hline
\end{tabular}

a Presentación en botella de $1 \mathrm{~L}$.

\footnotetext{
${ }^{2}$ En el ámbito empresarial se entrevistaron a gerentes y técnicos de almazaras; en el institucional, a técnicos del sector de la Administración y, finalmente, en el ámbito educativo, a investigadores de Universidad.

${ }^{3}$ Dada la gran disparidad de precios de los aceites según su procedencia (Castilla-La Mancha, nacional y extranjero), se ha considerado un rango medio de precios de venta en el que es posible encontrar distintos tipos de aceites (girasol, oliva y oliva virgen extra), en diferentes superficies comerciales del área metropolitana de Madrid.
} 
La especificación de la técnica de Análisis Conjunto parte de la hipótesis de que las preferencias de las personas entrevistadas, o su valoración global del aceite, se obtiene a partir de las puntuaciones individuales de cada atributo, de tal manera que la suma de dichas puntuaciones genera la valoración global (Steenkamp, 1987). Se partió de un modelo aditivo ya que explica, en casi todos los casos, un porcentaje muy elevado (entre el $80 \%$ ó $90 \%$ ) de la variación de la preferencia de los individuos (Hair et al., 1999). Su formulación se muestra en la siguiente ecuación:

$$
\begin{gathered}
\text { Valoración }=\beta_{0}+\sum_{i=1}^{3} \beta_{i} D_{1 i}+\sum_{j=1}^{3} \beta_{j} D_{2 j}+ \\
+\sum_{k=1}^{3} \beta_{k} D_{3 k}+\sum_{l=1}^{3} \beta_{l} D_{4 l}
\end{gathered}
$$

donde $\beta_{1 i}, \beta_{2 j}, \beta_{3 k}$ y $\beta_{41}$ son los coeficientes asociados a los niveles $i(i=1,2,3)$; $(j=1,2,3), k(k=1,2,3)$, y I $(I=1,2)$ de los atributos precio (1), tipo (2), origen (3) y sistema (4), respectivamente, y donde $D_{1}, D_{2 j}$, $D_{3 k}$ y $D_{4 l}$ son las variables ficticias por cada atributo, considerando los niveles de cada atributo como categóricos.

El resultado final del programa Conjoint permite estimar las utilidades parciales de cada uno de los atributos y la utilidad total de cada perfil. Con las utilidades parciales de cada persona entrevistada y para determinar la estructura de preferencias de los consumidores, se calculó la importancia relativa (I.R.) de los atributos de cada una de ellas, siendo max $U_{i}$ la utilidad máxima y min $U_{i}$, la utilidad mínima. Así, la proporción de rango asignada a cada atributo, sobre la variación de rangos total ( $\mathrm{Hal}$ brendt et al., 1991; Hair et al., 1999), es;

$$
I R(\%)=\frac{\max U_{i}-\min U_{i}}{\sum\left(\max U_{i}-\min U_{i}\right)} \times 100
$$

Posteriormente y en función de la importancia relativa de los atributos para cada consumidor, se realizó un análisis multivariante de segmentación de conglomerados de K-medias sobre la estructura de preferencias utilizando el algoritmo Quick Segmentation Analysis del programa estadístico SPSS 15.0 (SPSS, 2008).

A continuación se determinó las cuotas de mercado de los aceites más preferidos mediante simu- lación empleándose el modelo de máxima utilidad (MU) (Bretton-Clark, 1986). El modelo MU supone que el consumidor elige aquel producto que le proporciona la utilidad máxima, obteniéndose la cuota de mercado como la proporción de veces que cada producto propuesto es elegido como el más preferido de entre todos los encuestados.

Por último, para determinar la máxima disposición al pago (MDP) se utilizó la regresión logística. La regresión logística es una técnica multivariante por medio de la cual se pretende estudiar las relaciones de asociación entre una variable dicotómica y una o varias variables independientes (cuantitativas o categóricas). Con base en la regresión logística, el método de valoración contingente (MVC) consiste simplemente en preguntar a un grupo de personas cuánto estarían dispuestas a pagar para obtener un determinado bien, o como hicieron Bishop y Heberlein (1979), en plantear si a un precio determinado el consumidor adquiere o no un determinado bien. En la década de los ochenta del siglo pasado, creció con rapidez el interés por el MVC, siendo Hanemann (1984) el que estableció las bases teóricas para su posterior aplicación.

Según Hanemann (1984), existe una relación entre los modelos de respuesta dicotómica y la teoría de la maximización de la utilidad que permite calcular la disposición al pago por un bien como medida del bienestar de los individuos, partiendo de la consideración de que el consumidor conoce, con certeza, su función de utilidad inicial.

Para calcular la diferencia de precio entre el aceite ecológico y convencional, el consumidor responde a una serie de preguntas sobre la disposición al pago. Siguiendo a Gil et al. (2000) se opta por una forma mixta, con preguntas de tipo binario y preguntas con formato abierto (Anexo 2). La primera pregunta consiste en averiguar si el encuestado está dispuesto o no a pagar un determinado sobreprecio $(10 \%, 25 \%, 50 \%$ y $100 \%$ por encima del aceite convencional), estableciéndose de forma aleatoria y proporcional cuatro grupos para cada uno de los incrementos de precio. Dependiendo de si la respuesta a la pregunta anterior ha sido positiva o negativa, se le formula una nueva pregunta, en el sentido de que indique cuanto más estaría dispuesto a pagar, en el primer caso, y cuál sería el

Anexo 2

¿Estaría dispuesto a pagar un $(10 \%, 25 \%, 50 \%, 100 \%)$ más por un aceite ecológico que por otro convencional?

Si la respuesta es positiva, indique cuánto más

Si la respuesta es negativa, indique hasta cuánto estaría dispuesto a pagar más

\begin{tabular}{lcccc}
\hline $\begin{array}{c}\text { Tipo de aceite } \\
\text { ecológico }\end{array}$ & $\begin{array}{c}\text { Precio de referencia } \\
\text { (botella } \mathbf{~ L )}\end{array}$ & sí & No & ¿Cuánto? \\
\hline Oliva Virgen Extra & $5 €$ & & \\
Oliva & $4 €$ & & \\
Girasol & $1,2 €$ & & \\
\hline
\end{tabular}


máximo sobreprecio que estaría dispuesto a pagar en el segundo caso.

De acuerdo con Hanemann (1984), la MDP se calcula mediante la estimación de la siguiente función logarítmica:

$$
D_{i}=\frac{1}{1+\ell^{-\left(a+b A_{i}\right)}}+u_{i}
$$

donde $D_{i}$ es una variable dicotómica que toma el valor unitario si el consumidor está dispuesto a pagar el sobreprecio y cero en caso contrario, $A_{i}$ recoge los precios de partida ofrecidos a los encuestados $(10 \%, 25 \%, 50 \%$ y $100 \%)$, a y b son los parámetros a estimar y $u_{i}$ el término de error. A partir de la función logarítmica anterior se calcula la disposición a pagar media de la siguiente manera:

$$
\begin{aligned}
& E(M D P)=\int_{0}^{\infty}\left(1-G_{M D P}(A) d A\right)= \\
& =\int_{0}^{\infty}\left(1+\mathrm{e}^{-(a+b A)}\right)^{-1} d A=-a / b
\end{aligned}
$$

Las características socioeconómicas de los encuestados se muestran en la Tabla 3.

\section{RESULTADOS Y DISCUSIÓN}

\subsection{Estructura de preferencias del consumidor}

Los resultados de la estimación de las preferencias del consumidor respecto a un aceite se muestran en la Tabla 4.

Considerando la población total, los atributos del aceite de mayor a menor importancia relativa, son: el tipo $(41,09 \%)$, el origen $(33,35 \%)$, el precio $(25,35 \%)$ y el sistema de producción $(0,20 \%)$. Las utilidades calculadas de cada uno de los niveles de los atributos, muestran que los consumidores de aceite se decantan por aceites de oliva virgen extra, a ser posible de origen nacional, baratos y elaborados convencionalmente.

A partir de la importancia relativa concedida a los atributos se procedió a la segmentación de la población total, detectándose tres segmentos de consumidores que difieren en su estructura de preferencias de aceite. Un primer segmento, que agrupa al $47,3 \%$ de los consumidores, valora sobre todo el tipo de aceite; un segundo segmento, que agrupa al $29,4 \%$ de los consumidores, considera como atributo más importante el precio y finalmente, un tercer segmento, que agrupa al $23,3 \%$ de los consumidores, considera como atributo más importante el origen del aceite.

Tabla 3

\begin{tabular}{|c|c|c|c|}
\hline Característica & Niveles & Población ${ }^{\text {b }}$ & Muestra \\
\hline \multirow[t]{2}{*}{ Genero } & Mujer & 47,0 & 49,0 \\
\hline & Hombre & 53,0 & 51,0 \\
\hline \multirow[t]{5}{*}{ Edad (años) } & $18-24$ & 13,1 & 12,9 \\
\hline & $25-34$ & 21,5 & 21,2 \\
\hline & $35-49$ & 27,0 & 28,6 \\
\hline & $50-64$ & 19,6 & 19,0 \\
\hline & $>64$ & 18,8 & 18,3 \\
\hline \multirow[t]{3}{*}{ Estudios } & Elementales & 26,4 & 26,7 \\
\hline & Medios & 43,9 & 43,1 \\
\hline & Superiores & 29,7 & 30,2 \\
\hline \multirow[t]{6}{*}{ Ocupación } & Empresario & 7,7 & 6,4 \\
\hline & Empleado & 52,5 & 51,2 \\
\hline & Ama de casa & 11,6 & 13,8 \\
\hline & Estudiante & 5,8 & 10,0 \\
\hline & Jubilado & 11,2 & 13,8 \\
\hline & Otra & 11,2 & 4,8 \\
\hline \multirow[t]{5}{*}{ Renta } & $<900 €$ & 11,5 & 10,2 \\
\hline & de $900 €$ a $1500 €$ & 26,6 & 27,6 \\
\hline & de $1500 € a 2100 €$ & 16,6 & 32,2 \\
\hline & de $2100 € a 3000 €$ & 19,0 & 18,8 \\
\hline & $>3000 €$ & 26,3 & 10,2 \\
\hline \multirow[t]{2}{*}{ Hábitat } & Madrid capital & 73,7 & 75,7 \\
\hline & Área metropolitana & 26,3 & 24,3 \\
\hline
\end{tabular}

Características socioeconómicas de la población y de la muestra encuestada (\%)

${ }^{\mathrm{b}}$ Fuente: Elaboración propia a partir de datos del IECM (2006) e INE (2006). 
Tabla 4

Utilidades asignadas a los niveles de los atributos

\begin{tabular}{|c|c|c|c|c|c|c|c|c|}
\hline \multirow[t]{2}{*}{ Atributos y niveles } & \multicolumn{2}{|c|}{ Población total } & \multicolumn{2}{|c|}{$\begin{array}{c}\text { Segmento } 1 \\
(47,3 \%)^{c}\end{array}$} & \multicolumn{2}{|c|}{$\begin{array}{c}\text { Segmento } 2 \\
(29,4 \%)^{c}\end{array}$} & \multicolumn{2}{|c|}{$\begin{array}{c}\text { Segmento } 3 \\
(23,3 \%)^{c}\end{array}$} \\
\hline & IR (\%) & Util. & IR (\%) & Util. & IR (\%) & Util. & IR (\%) & Util. \\
\hline Precio*** & 25,35 & & 22,32 & & 40,00 & & 18,74 & \\
\hline $\begin{array}{l}1,20 € \\
4 € \\
5 €\end{array}$ & & $\begin{array}{r}1,316 \\
-0,694 \\
-0,622\end{array}$ & & $\begin{array}{r}1,347 \\
-0,729 \\
-0,618\end{array}$ & & $\begin{array}{r}1,638 \\
-0,712 \\
-0,926\end{array}$ & & $\begin{array}{r}0,849 \\
-0,604 \\
-0,246\end{array}$ \\
\hline Tipo*** & 41,09 & & 49,01 & & 37,49 & & 21,95 & \\
\hline $\begin{array}{l}\text { Oliva Virgen Extra } \\
\text { Oliva } \\
\text { Girasol }\end{array}$ & & $\begin{array}{r}1,332 \\
0,594 \\
-1,926 \\
\end{array}$ & & $\begin{array}{r}1,846 \\
0,865 \\
-2,712\end{array}$ & & $\begin{array}{r}1,044 \\
0,316 \\
-1,359\end{array}$ & & $\begin{array}{r}0,653 \\
0,397 \\
-1,049\end{array}$ \\
\hline Origen $^{\star \star \star}$ & 33,35 & & 27,22 & & 22,40 & & 56,80 & \\
\hline $\begin{array}{l}\text { Castilla-La Mancha } \\
\text { Nacional } \\
\text { Extranjero }\end{array}$ & & $\begin{array}{r}0,824 \\
0,910 \\
-1,734 \\
\end{array}$ & & $\begin{array}{r}0,822 \\
0,855 \\
-1,677 \\
\end{array}$ & & $\begin{array}{r}0,366 \\
0,535 \\
-0,901\end{array}$ & & $\begin{array}{c}1,407 \\
1,498 \\
-2,905\end{array}$ \\
\hline Sistema* & 0,20 & & 1,45 & & 0,11 & & 2,51 & \\
\hline $\begin{array}{l}\text { Convencional } \\
\text { Ecológico }\end{array}$ & & $\begin{array}{r}0,008 \\
-0,008\end{array}$ & & $\begin{array}{r}0,067 \\
-0,067\end{array}$ & & $\begin{array}{r}-0,004 \\
0,004\end{array}$ & & $\begin{array}{r}-0,097 \\
0,097\end{array}$ \\
\hline
\end{tabular}

${ }^{\mathrm{c}}$ Tamaño del segmento

${ }^{* * *} \mathrm{y}$ * Indican diferencias significativas con un error máximo de un $1 \%$ y un $10 \%$, respectivamente

IR = Importancia Relativa; Util. = Utilidad.

En el análisis estadístico efectuado, se han encontrado diferencias significativas entre los consumidores de aceite de los tres segmentos, en función de las importancias relativas de los atributos. El análisis de dichas diferencias revela que:

En el precio del aceite, el precio más bajo $(1,20 €)$ es valorado por encima de la media por los consumidores de los segmentos 1 y 2 , y por debajo, por los consumidores del segmento 3 . El precio de $4 €$, es valorado negativamente por los tres segmentos, fundamentalmente más por los consumidores que basan su decisión en el tipo que por los que basan su decisión en el precio o en el origen. Las principales diferencias aparecen entre los segmentos 2 y 3, confirmándose que los consumidores que se orientan por el atributo origen al comprar el aceite, conceden menos importancia a los precios altos que los consumidores que basan su decisión en el precio.

En el tipo de aceite, las diferencias más significativas aparecen entre los segmentos de consumidores 1 y 3 . Los consumidores del segmento 1 vaIoran más los aceites de oliva virgen extra y menos los aceites de oliva y los de girasol mientras que los consumidores de los segmentos 2 y 3 valoran por debajo de la media los aceites de oliva virgen extra y los de oliva y por encima, los de girasol.

En el origen del aceite, las principales diferencias aparecen entre los segmentos de consumidores 2 y 3. Los consumidores del segmento 3 valoran más el origen, conceden una utilidad por encima de la media a los aceites de Castilla-La
Mancha y a los nacionales. Sin embargo, los consumidores preocupados por el precio (segmento 2), valoran los aceites de Castilla-La Mancha y nacionales por debajo de la media. Los aceites extranjeros, pese a tener una utilidad negativa para todos los consumidores, son menos valorados por los que conceden una mayor importancia al origen del aceite que por aquellos que valoran fundamentalmente el precio.

En el sistema de producción, las diferencias significativas aparecen entre los segmentos de consumidores 2 y 3 y el segmento 1. Los consumidores de los segmentos 2 y 3 valoran por encima de la media los aceites procedentes de agricultura ecológica, mientras que el segmento 1 los valora por debajo.

\subsection{Simulación de cuotas de mercado}

Una vez analizadas las preferencias del consumidor por las que se detectó que el aceite más preferido era el aceite de oliva virgen extra, se evaluaron las posibilidades de distintos aceites de oliva virgen extra en función de su origen (de Castilla-La Mancha o del resto de España), precio y sistema de elaboración (convencional o ecológico), lo cual puede representar situaciones probables de competencia comercial.

Teniendo en cuenta estas restricciones, se definieron dos escenarios posibles con distintos tipos de aceites a los que se calculó por simulación su cuota de mercado mediante el método de máxima 
utilidad. En el primer escenario se analizaron las distintas posibilidades de éxito de ocho aceites y en el segundo, se eliminaron aquellos aceites menos preferidos por el conjunto de los tres segmentos de consumidores (Tabla 5).

Los resultados obtenidos en la Tabla 5 y para el Escenario I, muestran que las mayores cuotas de mercado se consiguen para los aceites nacionales distintos a los de Castilla-La Mancha.

En general, los consumidores de aceite de oliva virgen extra en el mercado de Madrid cuando lo eligen por su origen lo hacen de marcas nacionales y cuando lo hacen por su precio, por las marcas de Castilla-La Mancha. En este sentido, los consumidores de los segmentos 1 y 2 son los que muestran una mayor predisposición al consumo de estos aceites procedentes de Castilla-La Mancha y en especial, los del segmento 2 , que valoran especialmente los aceites ecológicos a bajo precio, lo cual puede constituir el segmento objetivo para los aceites de oliva producidos en Castilla-La Mancha.

En el Escenario II, una vez eliminados los aceites menos preferidos para el conjunto de los tres segmentos de consumidores, se analizaron cuatro posibilidades, de las cuales una correspondía a los aceites de oliva virgen extra, ecológicos, producidos en Castilla-La Mancha y los otros tres, al resto de España. En este segundo escenario, no siendo tan evidente cómo influye la variable precio en el consumo de aceite de oliva virgen extra cuando el origen del aceite es nacional, las ventajas comparativas para el aceite producido en Castilla-La Mancha $^{4}$ pasan por producir un aceite ecológico, lo cual podría llegar a suponer alrededor del $30 \%$ de la cuota de mercado de los aceites consumidos en el mercado de Madrid.

\subsection{Disposición máxima al pago por un aceite ecológico}

La disposición máxima al pago de los consumidores por un aceite ecológico (respecto a otro elaborado convencionalmente), sería de un sobreprecio del $13,1 \%$, que se corresponde con un aceite de oliva virgen extra (Tabla 6).

Tabla 5

Simulación de la cuota de mercado ${ }^{(\mathrm{d})}$ de aceite oliva virgen extra en función de las preferencias de los consumidores

\begin{tabular}{|c|c|c|c|c|c|c|}
\hline & Escenario/Sistema & $\begin{array}{c}\text { Precio } \\
(€ / L)\end{array}$ & Origen & $\begin{array}{c}\text { Segmento } 1 \\
\text { (Tipo) } \\
(\%)\end{array}$ & $\begin{array}{c}\text { Segmento } 2 \\
\text { (Precio) } \\
\text { (\%) }\end{array}$ & $\begin{array}{c}\text { Segmento } 3 \\
\text { (Origen) } \\
(\%)\end{array}$ \\
\hline \multirow[t]{8}{*}{ I } & Convencional & 4 & Castilla-La Mancha & 15,7 & 7,6 & 10,3 \\
\hline & Ecológico & 4 & Castilla-La Mancha & 12,0 & 21,1 & 4,5 \\
\hline & Convencional & 5 & Castilla-La Mancha & 16,5 & 7,0 & 8,2 \\
\hline & Ecológico & 5 & Castilla-La Mancha & 4,0 & 8,0 & 16,6 \\
\hline & Convencional & 4 & Nacional & 7,2 & 20,1 & 18,2 \\
\hline & Ecológico & 4 & Nacional & 15,3 & 9,9 & 6,6 \\
\hline & Convencional & 5 & Nacional & 17,3 & 13,6 & 19,2 \\
\hline & Ecológico & 5 & Nacional & 12,0 & 12,6 & 16,6 \\
\hline \multirow[t]{4}{*}{ II } & Ecológico & 4 & Castilla-La Mancha & 31,0 & 31,5 & 25,6 \\
\hline & Convencional & 4 & Nacional & 17,6 & 28,5 & 23,0 \\
\hline & Ecológico & 5 & Nacional & 18,8 & 19,8 & 24,7 \\
\hline & Convencional & 5 & Nacional & 32,6 & 20,2 & 26,7 \\
\hline
\end{tabular}

(d) Método: Máxima Utilidad (MU)

Tabla 6

Disposición máxima al pago de los consumidores por un aceite ecológico

\begin{tabular}{lcccccc}
\hline \multicolumn{1}{c}{ Tipo de aceite } & \multicolumn{2}{c}{$\begin{array}{c}\text { Segmento 1 } \\
\text { (Tipo) }\end{array}$} & $\begin{array}{c}\text { Segmento 2 } \\
\text { (Precio) }\end{array}$ & \multicolumn{2}{c}{$\begin{array}{c}\text { Segmento 3 } \\
\text { (Origen) }\end{array}$} \\
\cline { 2 - 7 } & $€$ & $\Delta$ (\%) & $€$ & $\Delta(\%)$ & $€$ & $\Delta(\%)$ \\
\hline Oliva Virgen Extra** $_{\text {Oliva }^{* \star *}}$ & 5,67 & 13,10 & 5,59 & 11,83 & 5,53 & 10,56 \\
Girasol $^{* * *}$ & 4,37 & 9,35 & 4,30 & 7,57 & 4,40 & 10,12 \\
\hline
\end{tabular}

** $\mathrm{y}^{* * *}$ Indican diferencias significativas con un error máximo de un $5 \%$ y un $1 \%$, respectivamente.

\footnotetext{
${ }^{4}$ En España, en el año 2007 la producción de aceite de oliva virgen fue de 1.258.100 t, de las cuales 85.667 t se produjeron en Castilla-La Mancha, lo cual supone el 6,81\% de la producción (JCCM, 2008).
} 
En general, los consumidores de aceite de oliva virgen extra están dispuestos a ofrecer unos incrementos mayores de dinero por un aceite de oliva virgen extra ecológico aún cuando el precio de referencia de los tres aceites evaluados es el más alto $(5 €)$. Esto puede deberse tal y como indica Sanz et al. (1997), a que se está pasando de un consumo de masas (en el que el consumidor orienta su compra en función del precio), hacia un consumo más racional (donde el consumidor está interesado por conocer las cualidades del producto que va a comprar). Respecto a los otros dos tipos de aceites (oliva y girasol), los porcentajes de incremento por los ecológicos son inversamente mayores respecto al precio de referencia del aceite convencional considerado.

\section{CONCLUSIONES}

En general, según las preferencias declaradas por los consumidores de aceite del área metropolitana de Madrid, los aceites se prefieren en función del tipo, origen, precio y sistema de elaboración, por este orden. En este sentido, el aceite más preferido es el de oliva virgen extra, nacional, barato y elaborado convencionalmente.

En función de sus preferencias individuales se han obtenido tres segmentos de consumidores de aceite. El primer segmento, que agrupa al 47,3\%, se caracteriza porque el atributo más preferido en sus decisiones de compra es el tipo; el segundo segmento, que agrupa al $29,4 \%$, tiene como atributo más preferido el precio $\mathrm{y}$, finalmente, el tercer segmento, que agrupa al $23,3 \%$, tiene como atributo más preferido el origen. En todos los casos, el atributo sistema de producción no parece resultar relevante en las decisiones de compra de los consumidores. En todo caso, es valorado positivamente por los consumidores de los segmentos que valoran fundamentalmente el precio y el origen.

Atendiendo a la cuota de mercado estimada, se confirma la preferencia por aceites de producción nacional y convencional. Aún así, parecen existir posibilidades de éxito para los aceites de oliva virgen extra producidos en Castilla-La Mancha en el mercado de Madrid, ya que los segmentos de consumidores que basan sus preferencias en el tipo y en el origen del aceite, valoran prácticamente por igual la procedencia de los aceites de oliva virgen extra de Castilla-La Mancha y del resto de España. En todo caso, parece existir una oportunidad de mercado para los aceites de oliva virgen extra ecológicos producidos en Castilla-La Mancha a precios competitivos.

En este sentido, la disposición máxima al pago de los consumidores por un aceite ecológico respecto a otro de las mismas características producido convencionalmente, es usualmente mayor para el aceite más caro (oliva virgen extra) que para los de menor precio, siendo el máximo sobreprecio que los consumidores están dispuestos a pagar del $13,1 \%$.
Por todo lo anterior, y para conseguir incrementar la cuota de mercado de los aceites de oliva producidos en Castilla-La Mancha sería necesario realizar estrategias de diferenciación.

La estrategia de diferenciación que en principio parece resultar más adecuada, es la de tipo y el origen del aceite, y complementarla, con la del sistema de producción ecológica.

De una manera específica, para los segmentos de consumidores 1 (tipo de aceite) y 3 (origen del aceite), es posible la realización de una estrategia comercial de comunicación conjunta en la que se ligara el aceite de oliva virgen extra con Castilla-La Mancha, conjugando la producción tradicional de la región con los beneficios saludables del consumo del aceite de oliva dentro de la llamada dieta mediterránea.

Para los consumidores del segmento 2, que basan sus decisiones de consumo básicamente en función del precio de compra del aceite, la estrategia comercial pasaría por ofrecer aceites de oliva virgen extra a precios más competitivos, para lo cual básicamente se debería de incidir en las características complementarias del producto en sí, tales como el tipo y material de botella, tapón, etiqueta, etc., para la reducción de costes y suplementariamente, realizar ofertas comerciales basadas principalmente en reducciones de precio por volumen de compra del consumidor (compra 3 y paga 2).

Adicionalmente a las anteriores estrategias de diferenciación, se podría añadir la de producción ecológica para así reforzar aún más el carácter de calidad de los aceites de oliva de Castilla-La Mancha.

Complementariamente a las estrategias de diferenciación empresarial, la Administración de Castilla-La Mancha podría realizar campañas genéricas de comunicación, dirigidas al mercado objetivo, en donde el cuerpo del mensaje pasaría por resaltar el nexo de unión entre la producción tradicional de aceite oliva (y en especial de oliva virgen extra) de Castilla-La Mancha y calidad.

En este sentido, la producción ecológica de aceite de oliva puede convertirse en un elemento de diferenciación esencial en el mercado del aceite de oliva y, dadas las características de producción del olivar en Castilla-La Mancha (en las que es relativamente fácil el paso de un olivar convencional a otro ecológico), constituir en el futuro próximo un elemento clave de competitividad empresarial. Por ello, sería de gran ayuda que desde la Administración de Castilla-La Mancha se pudiera promover no sólo campañas de comunicación sino, además, una posible declaración de Indicación Geográfica Protegida de Aceites de Oliva Ecológicos de CastillaLa Mancha.

\section{AGRADECIMIENTOS}

Los autores agradecen al Fondo Social Europeo y la Junta de Comunidades de Castilla-La Mancha 
la financiación del Proyecto de Investigación PBI080258 "Análisis comercial de la agricultura ecológica en Castilla-La Mancha", en el marco del Programa Operativo FSE 2007-2013.

\section{REFERENCIAS}

Aguirre MS, Aldamiz-Echevarria C, Charterina J, Vicente A. 2003. El consumidor ecológico. Un modelo de comportamiento a partir de la recopilación y análisis de la evidencia empírica. Distribución y Consumo 67, 4154.

Armstrong G, Farley H, Gray J, Durkin M. 2005. Marketing health-enhancing foods: implications from the dairy sector. Marketing Intelligence \& Planning 23, 705719.

Bernabéu R, Olmeda M. 2007. El consumidor de alimentos ecológicos en Castilla-La Mancha. Ediciones de la Universidad de Castilla-La Mancha. Cuenca.

Bishop RC, Heberlein TA. 1979. Measuring values of Extra-Market goods: Are Indirect Measures Biased? Am. J. of Agric. Economics 61, 926-930.

Braña T, Ferraces M J, Varela J. 1995. Diseño factorial incompleto. IV Simposium de Metodología de las Ciencias del Comportamiento. Murcia.

Bretton-Clark. 1986. Conjoint Designer and Conjoint Analyzer version 2.0. Bretton-Clark

Brugarolas M, Rivera LM. 2001. Comportamiento del consumidor valenciano ante los productos ecológicos e integrados. Rev. de Estudios Agrosociales y Pesqueros 192, 105-121.

Chang HS, Zepeda L. 2004. Demand for organic food: focus group discussions in Armidale. NSW. Working Paper Series in Agricultural and Resource Economics, 6. [En línea]: http://www.une.edu.au/febl/EconStud/wps.htm (Consulta de 15 de febrero de 2006).

De Boer J, Helms M, Aiking H. 2006. Protein consumption and sustainability: Diet diversity in EU-15. Ecological Economics 59, 267-274.

Gil JM, Soler F, Díez I, Sánchez M, Sanjuán AI, Ben Kaabia M, Gracia A. 2000. Potencial de mercado de los productos ecológicos en Aragón. Ed. Diputación General de Aragón. Zaragoza.

Green PE, Rao VR. 1971. Conjoint Measurement from Quantifying Judgemental Data. J. of Marketing Research 8, 355-363.

Hair JF, Anderson RE, Tatham RL, Black WC. 1999. Análisis multivariante. Prentice-Hall. Madrid.

Halbrendt CK, Wirth EF, Vaughn GF. 1991. Conjoint analysis of the Mid-Atlantic food-fish market for farm- raised hybrid stripted bass. Southtern J .of Agric. Economics, July, 155-163.

Hanemann WM. 1984. Welfare Evaluation in Contingent Valuation Experiments with Discrete Response. Am. J. of Agric. Economics 66, 332-341.

Instituto de Estadística de la Comunidad de Madrid (IECM) 2006. Estructura y evolución de la población. [En línea]: http://www.madrid.org/desvan/almudena/arbolalmudenalista.jsp (Consulta realizada el 11 de septiembre de 2006).

Junta de Comunidades de Castilla-La Mancha (JCCM) 2008. Cifras del sector agrario. [En línea]: http://www.jccm.es/agricul/paginas/agricultura-ganaderia/cifras/aceite.htm (Consulta realizada el 20 de abril de 2009).

Kirk J. 1982. Experimental design: Procedures for the behavioural sciences. Brooks-Cole Co. 2nd ed. Monterrey, CA.

Mann S. 2003. Why organic food in Germany is a merit good. Food Policy 28, 459-469.

Ministerio de Agricultura, Pesca y Alimentación (MAPA) 2007. La alimentación en España 2006. [En línea]: http://www.mapa.es/alimentacion/pags/consumo/2006/panel-06.pdf (Consulta realizada el 19 de marzo de 2007).

Ministerio de Agricultura, Pesca y Alimentación (MAPA) 2008. Estadísticas 2007 Agricultura Ecológica. España. [En línea]: http://www.mapa.es/alimentacion/pags/ecologica/pdf/2007.pdf (Consulta realizada el 5 de febrero de 2009).

Noomene R. 2005. Actitudes del consumidor español ante los alimentos modificados genéticamente (OGM). Tesis Máster en Ciencias (no publicada). Instituto Agronómico Mediterráneo. Zaragoza.

Rivera LM. 1989. Marketing para las Pymes agrarias $y$ alimentarias. MAPA-AEDOS. Madrid

Sanjuán Al, Sánchez M, Gil JM, Gracia A, Soler F. 2003. Brakes to organic market enlargement in Spain: consumers' and retailers' attitudes and willingness to pay. Int. J. Consumer Studies 27, 134-144.

Sanz, F.J.; Rodríguez-Zúñiga, M. y Mili, S. (1997). Estrategias competitivas ante la globalización de los intercambios comerciales: la cadena del aceite de oliva en España. Instituto de Economía y Geografía (C.S.I.C.). Madrid.

SPSS Inc. 2008. SPSS Categories. Version 15.0. Chicago.

Steenkamp JB. 1987. Conjoint measurement in ham quality evaluation. J. of Agric. Economics 38, 473-480.

Recibido: 4/5/09 Aceptado: 3/6/09 